\title{
Instability of a homogeneous flow from a lumped source in the presence of special boundary conditions on a free surface
}

\author{
Anastasia Shmyrova* and Andrey Shmyrov \\ Institute of Continuous Media Mechanics UB RAS, Perm, Russia
}

\begin{abstract}
In this work, experimental studies were carried out to investigate the structure of a surface flow and to understand potential mechanisms leading to the formation of this type instability. The surface flow was generated by feeding water through three sources: a lumped source with free upper boundary, a slot gap, and a lumped source for inducing a capillary-driven Marangoni flow. For flow visualization, a traditional light knife technique was used. The application of a method of spatial separation of the water volume into two isolated parts whose common surface remained unchanged and the realization of a reverse situation with a divided surface made it possible to study in detail the surface flow structure and to determine the conditions for the appearance of such hydrodynamic instability. It is shown that the formation of a vortex flow is caused by the interaction between the coordinate of the flow homogeneous along the transverse flow and the layer of a surface-active substance adsorbed at the interface. The obtained experimental results demonstrate the importance of setting different boundary conditions for potential and vortex velocity components of a convective flow in the region near the interface occupied by a surfactant.
\end{abstract}

\section{Introduction}

Multiphase media are currently the subject of extensive investigation. They have found wide application in various technological processes (production of emulsions and foams), in chemical and biochemical technologies (control of emulsification and wetting processes, increase of colloidal medium stability, etc.), and in biology and medicine. However, their use in the presence of surfaceactive molecules (surfactant) is complicated because of a monomolecular film formed at their interface due to adsorption-desorption processes. The adsorbed layer observed in this case exhibits the rheological characteristics different from those of the volume phase: surface dilatation and shear elasticity, and surface dilatation and shear viscosity. The situation becomes even more complicated when the convective flows of different nature are generated in the system. The point is that there is a reciprocal relationship between the fluid velocity fields and the surfactant concentration at the interface. This produces a variety of effects, the study of which is a challenge but it may open new technological possibilities.

Differences in the properties of surface and volume phases can play a decisive role in solving the problem of the motion of a gas bubble or a drop of one liquid in another and the motion of a drop in a viscous gaseous phase. At the moment, analytical solutions to such problems exist for a small number of simple model approximations [1, 2]. However, despite their seeming simplicity, these problems do not have an analytical solution even in an axisymmetric formulation.

In [3-6] the results of theoretical and numerical studies on flows around a spherical bubble floating in the liquid containing a slightly soluble surfactant are presented. It was shown that the molecules of surfactants adsorbed at the interface change boundary conditions, and the liquid flow around the bubble causes the redistribution of surfactant molecules toward the backward pole, where they form a stagnant cap. In our opinion, on the surface of the stagnant cap there occurs a region with a vortex flow analogous to that observed on the flat surface in $[7,8]$. It was shown that, in this region with the volume flow, the uniform flow on the surface lost its stability and was converted into the vortex flow. The dimensions of vortices observed experimentally directly depended on the intensity of the main flow and the concentration of surfactants in the system. Investigation of the mechanism of viscous entrainment of insoluble surfactant molecules by the moving liquid using modern optical methods shows that the so-called Reynolds edge is formed on the boundary of the homogeneous flow with a surfactant film on the surface of the water and in the region occupied by the surfactant (the flow with vertical vorticity) [8]. The vortex motion of the liquid was also observed in the bulk phase located under the adsorbed monolayer. All the above-mentioned studies were carried out in long rectangular channels.

Based on the geometry of the system, it can be concluded that a possible cause of the formation of vortices at the surface of water is a sticking condition on the side wall of the channel. The transition to a cylindrical coordinate system would have made it possible to exclude the appearance of this effect and to predict the preservation of flow uniformity at the entire interface. However, it was shown in $[9,10]$ that the uniformity of azimuthal flows in

* Corresponding author: lutsik@icmm.ru 

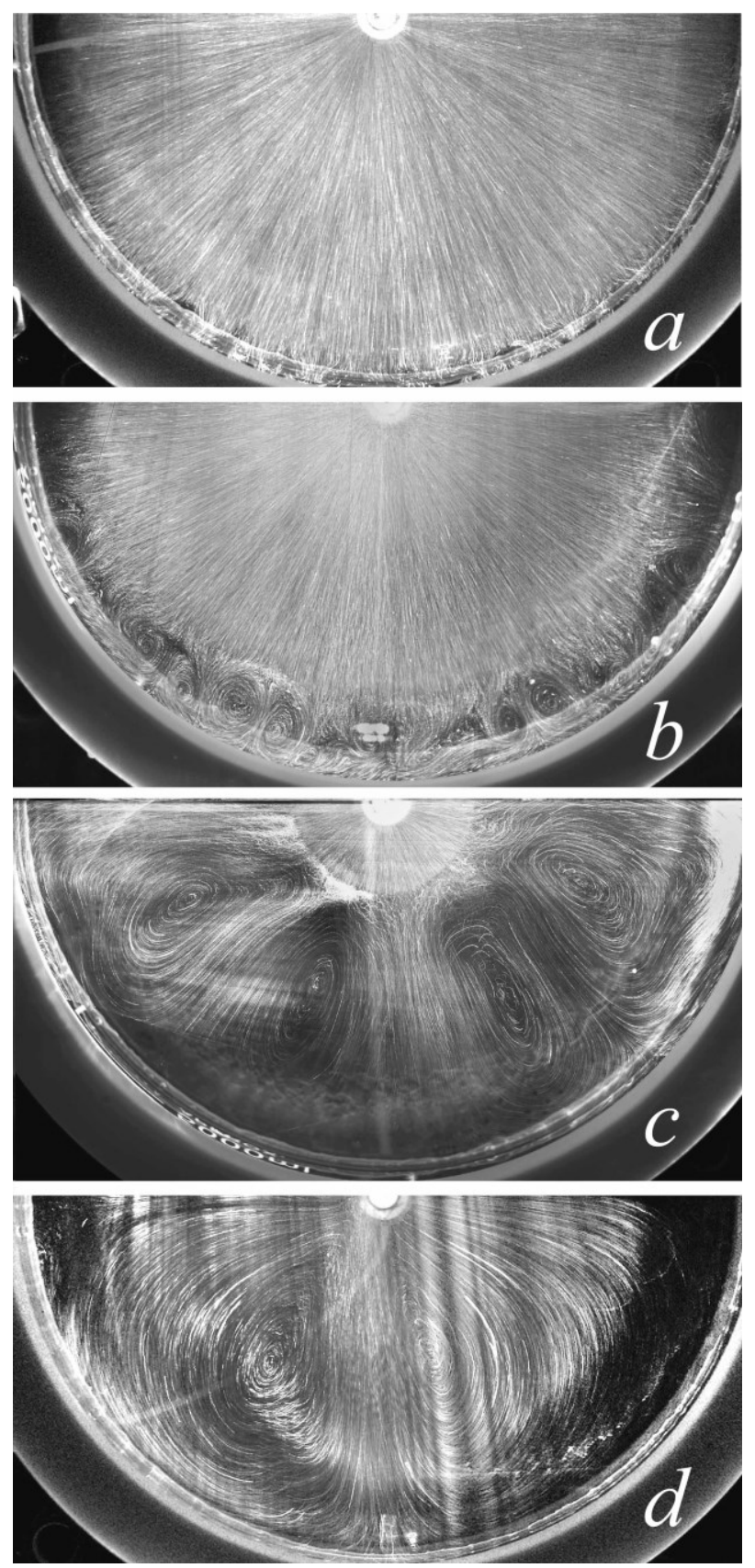

Fig. 1. Characteristic patterns of the surface flow structures obtained with increase in the surface density of the impurity and/or with decrease in the flow intensity.

the presence of the adsorbed surfactant layer is observed on the liquid surface area in which the vector velocity is directed against the main movement. This provides an indirect indication of the generation of a complex multivortex flow at the interface.

The survey of publications in this area should also include the works in which the fluid motion was not generated by volumetric forces; it was excited by capillary forces that occurred when a surface tension gradient was applied to the surface. In the studies on the thermal and concentration-capillary Marangoni convection from a lumped source in the systems with an interface containing surfactant molecules [11-18], it was shown that the radial flow was converted into the vortex

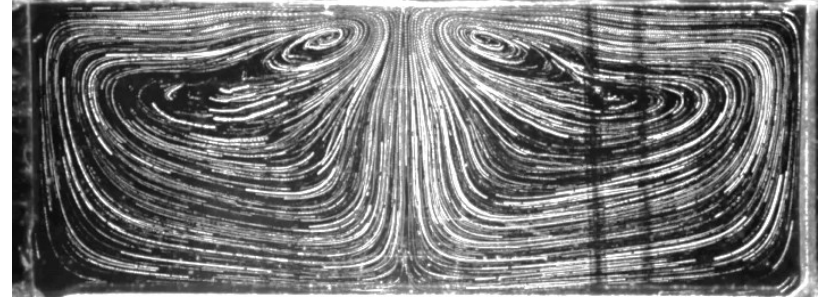

Fig. 2. Characteristic patterns of the volume flow structure obtained with thermocapillary flow.

flow irrespective of the nature of capillary forces generating the fluid motion on the surface occupied by the adsorbed surfactant film. The evolution of the characteristic flow structures is shown in Fig. 1 [18].

While the experimental and numerical results are described in detail, no physical explanation exists for the formation of a vortex flow on the surface of liquids. Moreover, the connection between the considered type of instability and the volumetric flow shape and intensity, whose role in the vortex flow problem is significant, should be elucidated. This phenomenon was partially clarified and explained in [19], in which the interaction of the thermocapillary flow from the lumped immaterial heat source with to the surface phase of surfactant molecules in the Hele-Shaw cell was investigated. The experiments showed that at specified control parameters the thermocapillary flow compresses the surfactant film toward the periphery of the cell, which leads to the formation of the region free of surfactant molecules near the source. At the free interface, the fluid velocity reaches its maximum, which indicates the development of intense Marangoni convection. Part of the surface occupied by the film restricts free movement and so the surface flow dives under the stagnant zone and forms in the volume convective shafts whose centers are pressed against the source (Fig. 2). The $2 \mathrm{D}$ thermal problem is a simplified version of a more complex three-dimensional problem, the solution of which is the subject of this paper.

\section{Experimental setup}

Investigation of the conditions for vortex flow formation in hydrodynamic systems with liquid-gas interfaces was conducted in cylindrical cuvette 1 shown in Fig. 3. The cuvette was made of durable transparent glass (diameter $179 \mathrm{~mm}$, and height $55 \mathrm{~mm}$ ). During the experiment, the cuvette was filled with water and installed on an alignment rack 2. The rack was placed on the Thorlabs vibration isolation optical table. The flow was visualized with a conventional light knife technique. The light knife, which was produced by laser 3 (wavelength $532 \mathrm{~nm}$ and power $200 \mathrm{~mW}$ ) and a system of lenses 4, traveled along the fluid surface. It illuminated the lightdiffusing particles (neutral-buoyancy hollow glass spheres) which were added into the working fluid. In the experiment, the tracers moving in the surface layer allowed us to visualize the flow structure and to observe its time variation. The typical flow patterns were track images obtained with the help of a highly sensitive video camera videoscan 5 with 11 Mpixel resolution. 


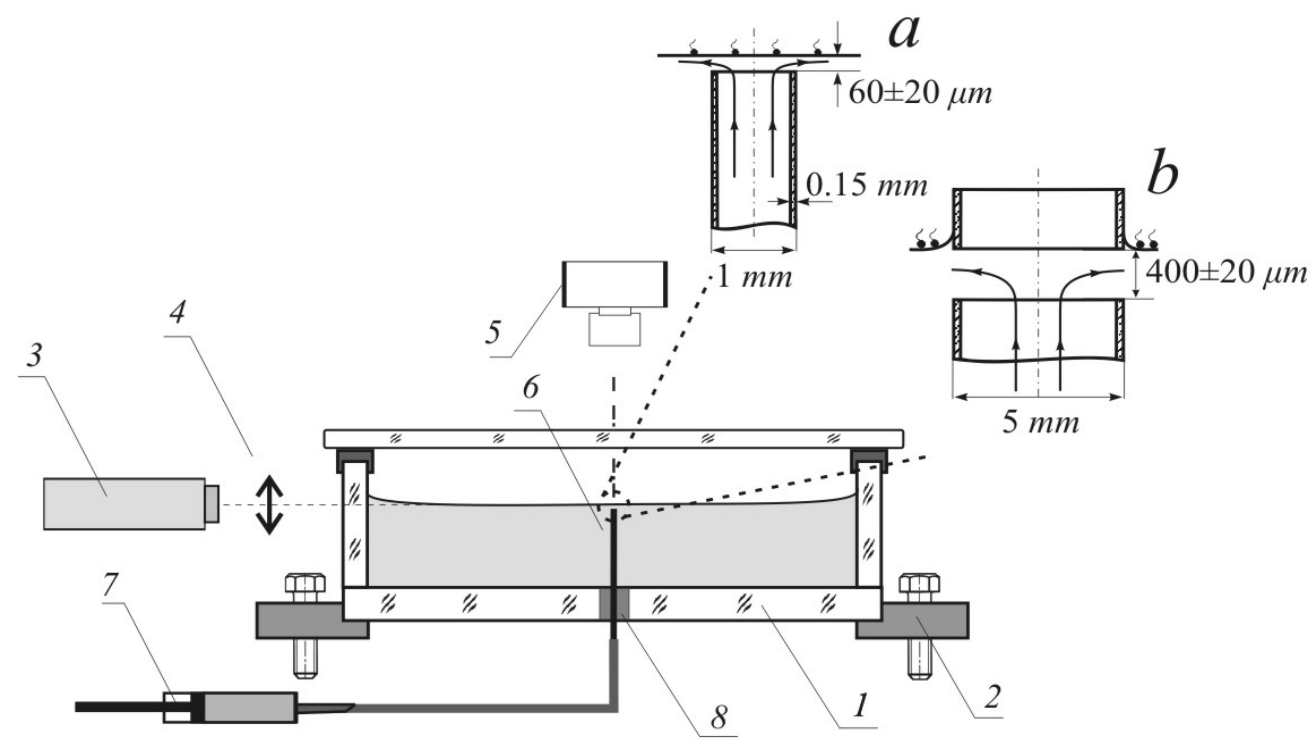

Fig. 3. Scheme of the experimental setup: 1 - cuvette, 2 - alignment rack, 3 - laser, 4 - lenses system, 5 - video camera, 6 - source, 7 - vessel, 8 - fluoroplastic insert.

The camera recorded the motion of particles with sizes from 5 microns and higher, and this improved the quality of track images. Shooting with a shutter speed $100 \mu$ s to $10 \mathrm{~s}$ was carried out depending on the flow intensity. The camera was placed above the experimental cell to capture the entire experimental area. In order to take a close-up picture of the flow near the source, the camera position can be changed. The camera was controlled by a computer, where further image processing was carried out. The cuvette was covered with a glass plate to avoid the penetration of impurities from the room, to reduce the evaporation of liquid from the cuvette and to decrease the influence of random air currents.

As water has the largest surface tension among ordinary liquids (excluding melts of metals and salts), one can take different surfactants to study the surface activity of the adsorbed surfactant layer in a wide range of physical and chemical parameters. On the other hand, working with water required more laborious work on preparation of the experimental setup. We used additional procedures aimed at obtaining a "zero" surface (surface without molecules of other substances). A three-stage Aquaphor with reverse osmosis filter was used to produce highly purified water. It removed bacteria, viruses, hardness salts, nitrates, nitrites and mechanical impurities in size up to $0.5 \mathrm{~nm}$. After that, the water was pumped from a pre-deioniser with the Bi-distiller Cyclon. At the output, we obtained ultrapure water with a conductivity of less than $0.1 \mu \mathrm{S} / \mathrm{cm}$ and $\mathrm{pH}$ of 5.6-6.0. During the research, all details of the experimental setup were cleaned using a detergent which was then removed under running water. Then the cuvette was rinsed with distilled water and, in the final stage, with water of high purity degree. The particles were also preliminarily cleaned. For this purpose they were sequentially washed with acetone, isopropyl alcohol, and, in the final stage, with high-purity water. At each stage, the particles were separated by gravitational sedimentation in the resting fluids. The particles with neutral buoyancy were deleted. It made possible to achieve a uniform distribution of tracers in a finite volume and to exclude the lighter particles film formation on the surface during the experiment. The preparatory procedures reduce, but not totally eliminate, the entry surfactant onto the working surface. To remove the remaining ones, the barrier-cleaning method was used. The cuvette was filled with a slight overflow for obtaining a convex meniscus. To this end, the edge of the cuvette was made from a hydrophobic material (fluoroplastic). Since surface-active impurities reduce the surface energy of the system, after a time their molecules are adsorbed onto the water surface from its thickness. The collection of random surfactant molecules from the water surface was carried out with the help of a polyacetal barrier. It is a hydrophilic material that, upon contact with water, is wetted, and a concave meniscus is formed at the waterbarrier boundary. Moving along the surface, the barrier collects ahead of itself surface-active impurity molecules. Then it is removed using an aspirator, and the impurityfree surface is formed behind the barrier. The purity of the water surface was monitored using two barriers and a Wilhelmy plate suspended from the high-sensitivity scales (KSV Instruments Ltd). The plate was partially submerged in water between the separated barriers. The surface was considered to be clean when the balance drift was less than $0.2 \mathrm{dyn} / \mathrm{cm}$ with the $90 \%$ area reduction. In the case when the change in surface tension exceeded the minimum allowable value, the cleaning procedure of the entire system was repeated again.

After cleaning, the liquid level dropped to the level of the light knife (approximately to the middle of the cuvette height). A surface azimuthally homogeneous flow was generated by feeding either a weakly concentrated (up to $10 \%$ of the mass concentration) of ethyl alcohol solution or water through the lumped sources 6 of different geometry ( $a, b$ types in Fig. 3). The results of the experimental work by D.G. Suciu [20] showed that the ethyl alcohol molecules are not absorbed at the interface, which may affect the concentration- 
capillary flow stability. The lumped source of the first type (6a, Fig. 3) was a thin (outer diameter of $1 \mathrm{~mm}$ ) steel tube, the upper section of which was as close as possible to the water surface. The gap thickness $h$ between the source and the liquid surface in the experiments was $60 \pm 20 \mu \mathrm{m}$ at $\mathrm{P}=0.95$. The gap $(\mathrm{h}=400 \pm 20 \mu \mathrm{m} \mathrm{P}=0.95)$ between two coaxial cylinders with diameter of $5 \mathrm{~mm}$ acted as a source of the second type ( $6 b$, Fig. 3$)$. The cylinders were attached to a $1 \mathrm{~mm}$ capillary and brought to the water surface until the gasliquid interface reached the gap between them. To supply liquid through the sources, the opposite end of this capillary was withdrawn from the cell and connected to the communicating vessel 7 by a flexible hose. Pumping of the weakly concentrated aqueous solution of ethyl alcohol or highly purified water was carried out using pump. Application if this pump made it possible to vary the mass flow of liquids in the range
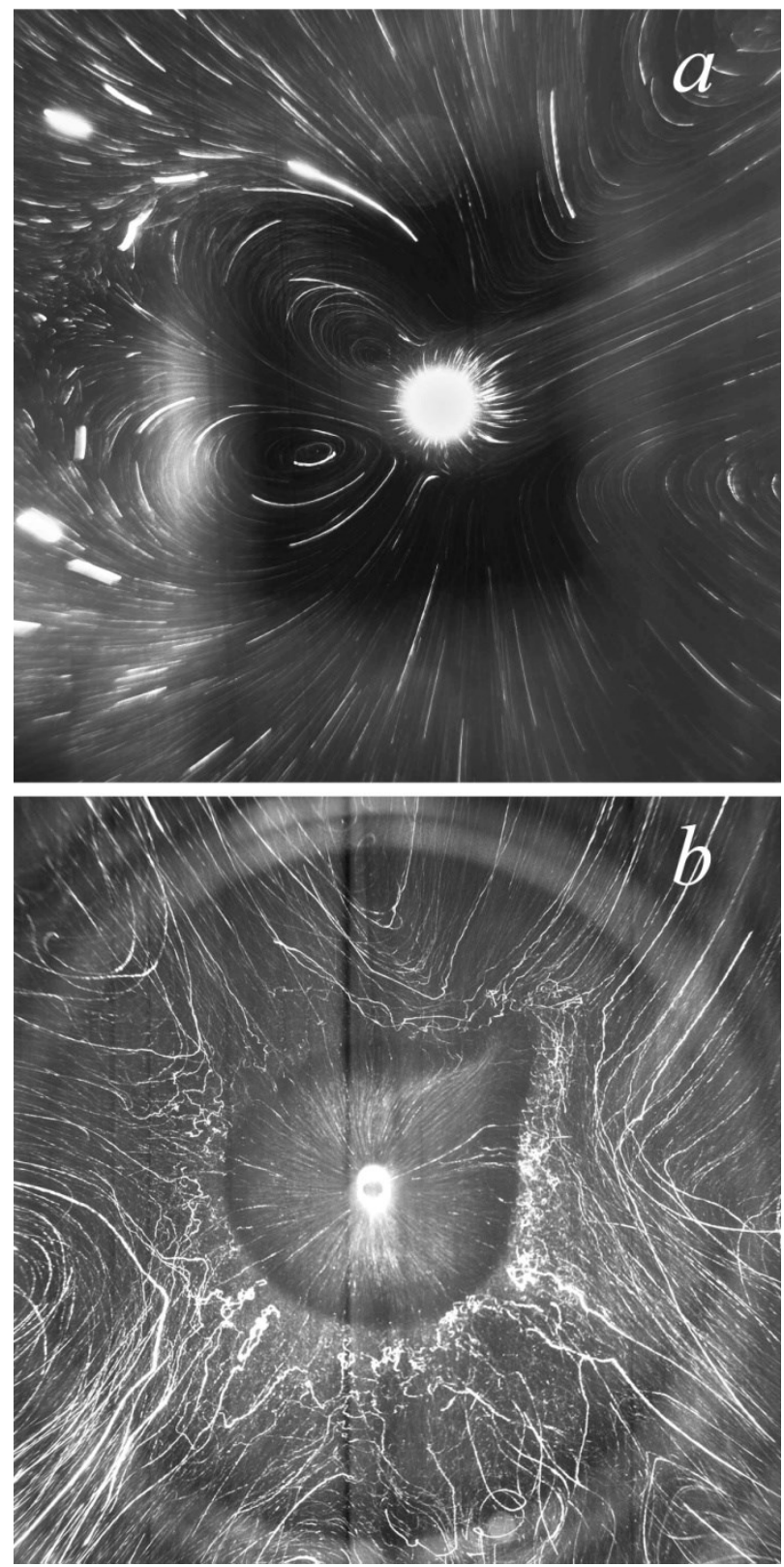

Fig. 4. The structure formation on azimuthal inhomogeneity of the surface flow.
$0.16 \cdot 10^{-2} \div 1.57 \mathrm{~g} / \mathrm{s}$. To avoid the leakage of liquid, the opening in the base of the cuvette was sealed with a fluoroplastic insert 8 . The difference in the configuration of the sources and the use of various liquids made it possible to realize capillary flows with shear stress of $3 \div 29 \mathrm{dyn} / \mathrm{cm}$ and volumetric flows of intensity of $0.05 \div 35 \mathrm{dyn} / \mathrm{cm}$.

The temperature of the liquids was $25.0 \pm 0.5^{\circ} \mathrm{C}$.

\section{Results and discussion}

Experimental results have demonstrated the necessity to do fine adjustment of the source position with respect to the liquid interface. It is shown that even a slight deviation of the supply capillary from the perpendicular and non-parallelism of the source boundary to the liquid surface lead to the formation of the azimuthal inhomogeneity of the flow (Fig. 4).

The most sensitive to such deviations was the displacing flow from the first type source (Fig. 4a). Unlike the concentration-capillary flow, the spreading of water cannot ensure a uniform motion of the fluid along the azimuth. With increasing flow rate, the effect only increased and a jet was formed on the liquid surface. This jet specified the direction of the fluid motion, which finally led to the violation of the vortex structure symmetry. When the ethyl alcohol solution was delivered to the water surface, the azimuthal homogeneity of the concentration-capillary flow formed automatically due to the potential nature of the capillary effect. However, some inhomogeneities can also be formed for this type of the flow (Fig. 4b). In order to minimize the described effect, at the stage of preparation of the experimental setup, the maximum possible adjustment of the source position relative to the interface was carried out. To this end, a laser ray adjusting screws 2 (Fig. 3) and a thin glass plate of small dimensions was uses. After cleaning, the partially filled with water cuvette was placed on a stand with adjusting screws. After preliminary equalization, a small glass plate was placed on the horizontal section of the source. An additional laser ray was directed perpendicular to it. One part of the ray was reflected from the surface of the plate, the second, passing through it, reflected from the surface of the water. Then two images of two reflected rays superposed with the help of adjusting screws 2 . This provided the alignment of source and liquid surfaces with an accuracy of several angular seconds. A syringe pump was used to minimize fluid pulsations supplying through the source. Apart from the above described procedure, the surfaces of the slit gap were smoothed and their alignment and the slit gap size were controlled using a Zeiss SteREO DiscoveryV12 microscope.

The described measures have made it possible to reduce the cumulative jet effect and to obtain the most uniform liquid flow along the azimuth. The symmetrical track images of the vortex flow obtained through the first-type source at maximum possible volume flow velocity provide further evidence of this fact (Fig. $5 b$ ). Analysis of the experimental results has revealed that four is the maximum possible number of vortices 

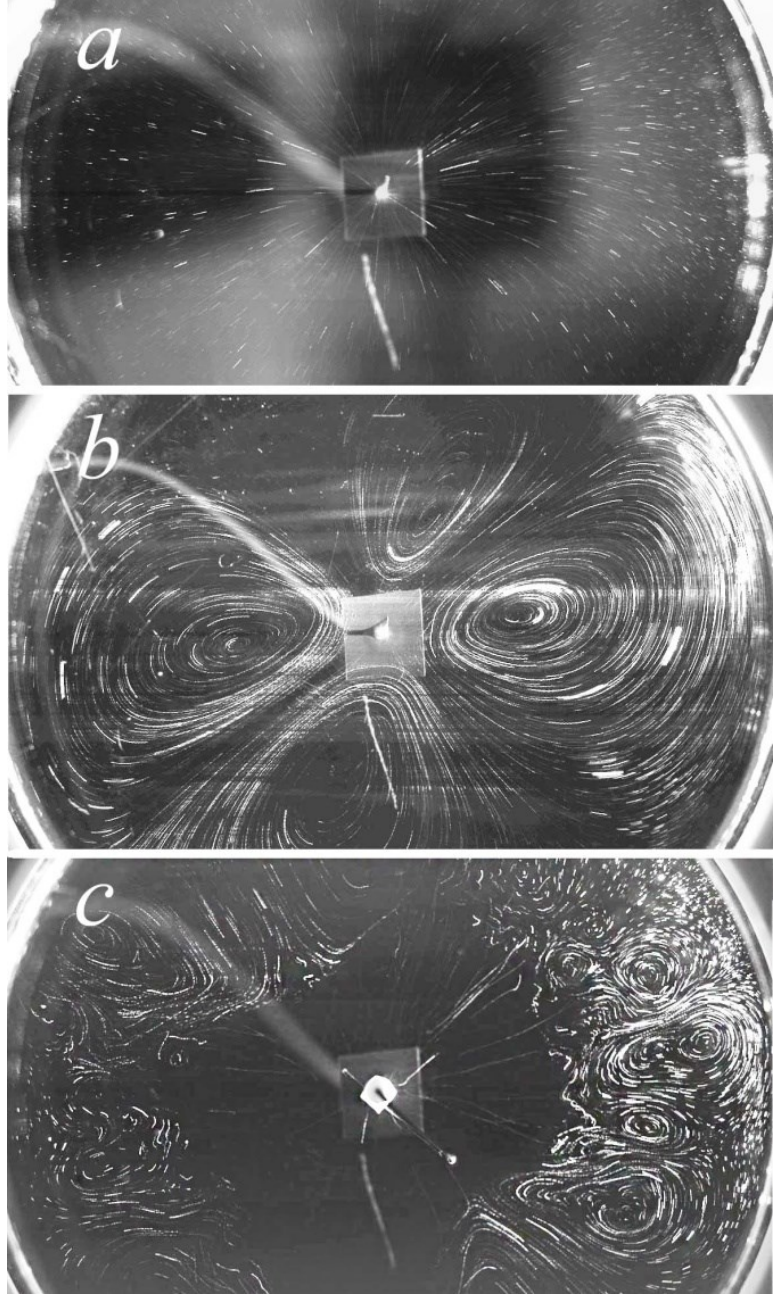

Fig. 5. The track images obtained with hydrodynamic flow on the clean surface $(a)$ and on the surface covered with surfactant molecules from the lamped source $(b)$ and gap $(c)$.

generated by the first type source; further increase in the flow rate of the liquid passing through the capillary results produces jet flowing perpendicularly to the surface. The transition from the capillary with free upper boundary to the slit (second type source) makes it possible to increase the velocity of the volume flow. Therefore, the number of vortices increases and a zone free from surfactant molecules is formed near the source (Fig. $5 c$ ). The track images for the hydrodynamic flow (Fig. 5) are similar to those obtained for the alcohol solutions (Fig. 1). It indicates that, regardless of the source type and the nature of the generated flow in the presence of surfactant molecules, the uniform flow along the radius of the clean surface (Fig. 1a, Fig. $4 a$ ) is converted to the vortex flow consisting of vortices periodically located along the angular coordinate (Fig. $1 b-d$, Fig. $5 b, c$ ). The number of vortices and the size of the zone free from surfactant molecules depend on the force exerted on the surface layer.

Registration of large velocity gradients is another important result. Our experimental study revealed one more feature of the instability formed in the systems with interfaces. The flow structure was found to vary qualitatively in a thin near-surface layer: from the vortex

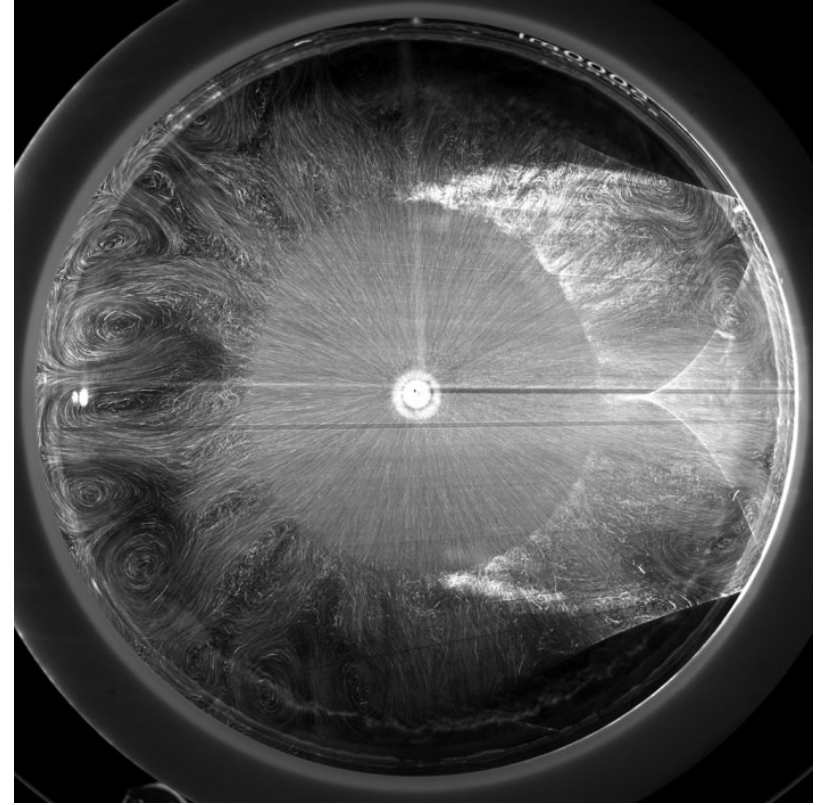

Fig. 6. The flow structure obtained with solutocapillary flow (inclined laser knife).

system at the surface to the system of jets at a depth of 3$5 \mathrm{~mm}$ under it (Fig. 6).

The flow structure on the surface is on the right side of the image, and the flow structure under the surface is on the left side of the image. By analyzing the right part of the image, one can see that the boundary from the radial flow to the compressed surfactant film is a line. On the left side of the image this line is more diffused and finally is replaced by a zone of finite width. Differences can also be clearly seen in the structure of the flow in the transition zone from the radial to the eddy current. In the near-surface region the axisymmetric radial flow dives under the film and generates an azimuthal inhomogeneity in the form of jets, of which one is the source inducing the formation of two vortices (the left part of the image). The same area on the right side of the image does not have such a clear radial structure, but represents a zone of tracks that do not create a definite picture of the current. However, on the periphery of the cuvette, as well as on the left, the zone of the multivortex flow is easily read. Thus, it can be concluded that the flow on the surface and directly below it, having a similar structure, simultaneously has significant differences.

Large velocity gradients along the normal to the surface, the refraction of the laser beam on the meniscus caused by the contact of the liquid with the walls of the cuvette, and the final thickness of the laser knife $(\sim 0.1$ $0.2 \mathrm{~mm}$ ) lead to the appearance of crisscross tracks and the summation of data from different depths. The latter makes it difficult to analyze the image with small free surface dimensions concentrated near the sources. In order to determine the structure of the flow directly on the surface and to fix the critical values of the control parameters at which the formation of polynya is formed, talc particles were used as floating tracers. Talc was precalcined at a high temperature to remove surface-active organic impurities. Then it was applied to a prepared 


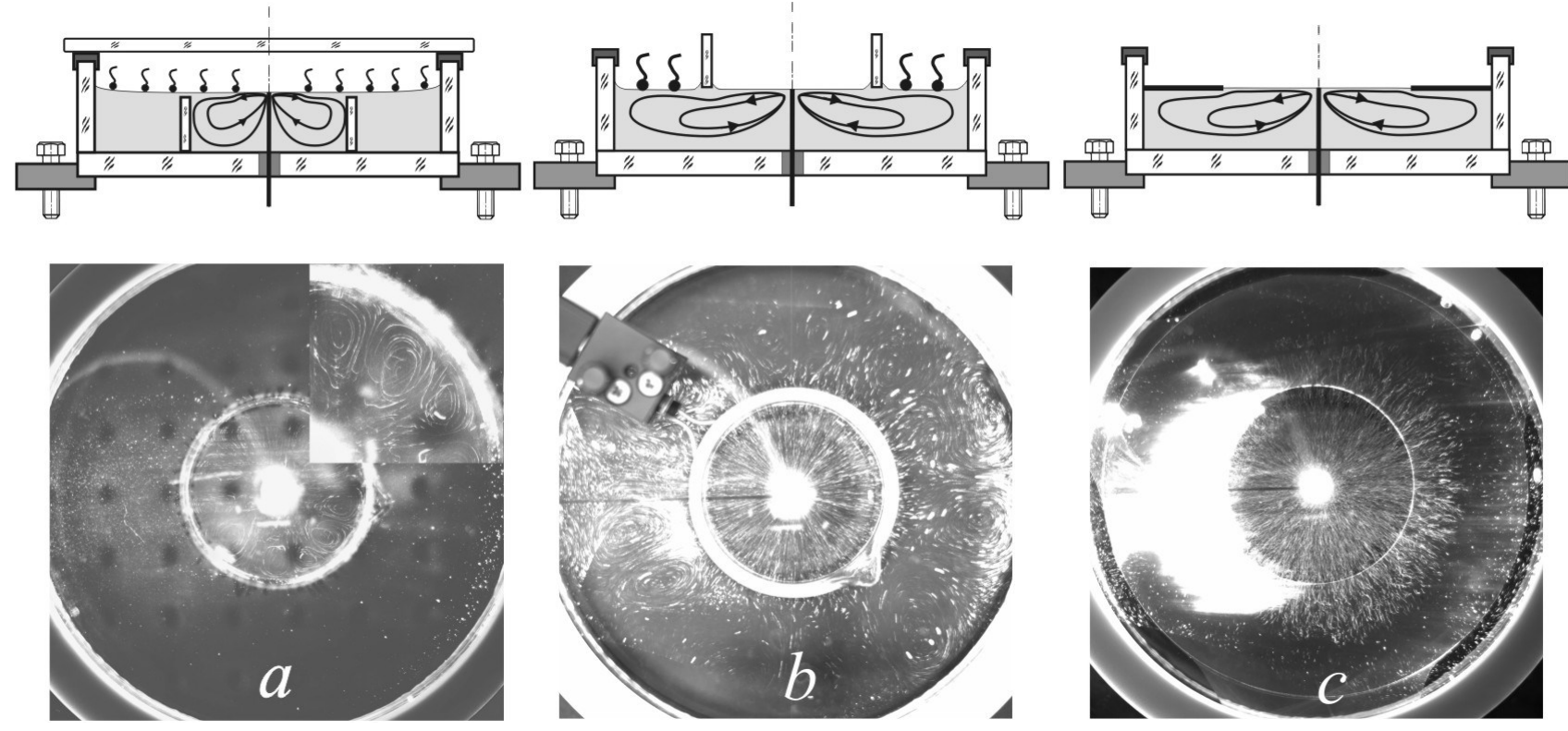

Fig. 7. The vortex flow formation on the system with divided volume $(a)$, divided surface $(b)$, a solid surface $(c)$.

aqueous surface filled with oleic acid molecules. Since mechanically the talc powder is practically impossible to apply on the surface, a miniature vortex chamber similar to the cyclonic chambers of modern vacuum cleaners was used to distribute it evenly. The compressed air was blown through a miniature vortex chamber partially filled with talc. The talc particles evenly filled the airflow and, leaving the chamber, settled on the surface of the water. Talc, unlike light-scattering particles in the bulk of a liquid, does not penetrate the interior of the system and is bound to an adsorbed surfactant layer. When the source was switched on, the surface tracers completely repeated the motion of the film, which was shifted by the flow and formed a system of vortices leaving the region near the source free of any particles (Fig. 5c). The latter made it possible to avoid discrepancies in the decoding of track images.

To determine the physical nature of the vortex flow and its connection with the volumetric flow in a number of experiments, the volume of water in the cuvette was divided into two parts isolated from each other by means of an annular screen with preservation of the common surface (Fig. 7a). The reverse configuration of the separation of the surface was also realized (Fig. $7 b$ ).

In the first case, an annular barrier of smaller radius was placed in the prepared cuvette filled with water. The liquid level in the cuvette was such that the gap between the free boundary of the liquid and the upper edge of the liner was of the order of $1 \mathrm{~mm}$. The barrier separated the volumes of the liquid save the free movement of the liquid on the entire surface. Experiments have shown that the absence of the bulk flow under the zone occupied by a surfactant leads to the impossibility of forming a vortex structure both in the volume and on the water surface (Fig. 7a). In the opposite situation, when the annular barrier was introduced from the gas phase, touching the surface of the liquid (Fig. 7b), the liner divided the surface into two coaxial zones that did not communicate with each other. The total volume of the liquid remained completely mobile. The presence of a surfactant film on the external surface led to the formation of a vortex flow, both in the bulk and on the surface. In this case, a radial flow was observed on the surface free from surfactant molecules inside the liner, which remained stable even if the coaxiality of the barrier arrangement was significantly violated. The considered experiments show that the formation of a multivortex flow is associated with the instability of the radial flow propagating below the surface of the stagnant zone. And an important role in this is played by the mobility of the surface phase. The experiment with a solid surface illustrates this assumption (Fig. 7c). A thin transparent film in the form of a ring was placed on the cleaned water surface. A source was located in the center of it. The radial flow from the source reaching the boundary of the solid film dived under it and retracted into the volume retaining its axial symmetry. The radial structure of the flow under the fixed surface was preserved even with further fluid motion in the volume.

\section{Conclusion}

The experimental study of three-dimensional hydrodynamic instability demonstrates the lack of an assumption about the axisymmetry of solutions of theoretical and numerical problems of similar formulation. A series of experiments on separation of the contributions of surface and bulk flows in the problem studying the interaction between the homogeneous flow from a lumped source and the adsorbed surfactant layer in a cylindrical cuvette showed that the formation of a vortex motion in a stagnant zone at the interface is associated with the rearrangement of a volumetric flow directly under the surfactant film. The flow reconstruction can be attributed to the fact that the surfactant film is immovable only in relation to the fluid motion causing its compression, unlike a really solid surface. The volumetric flow is formed when the surface 
current, encountering the boundary of the stagnant zone, dives under it, as under a solid surface.

Depending on the type of surfactant molecules, the adsorbed layer located in the stagnant zone responds differently to the applied viscous stresses.

In the case when the tangential stress field is potential and tends to compress the monolayer, such rheological surface characteristics as dilatational elasticity and viscosity counteract it. If the field of viscous tangential stresses applied to the liquid surface is predominantly of shear character, then only the surface shear viscosity offers resistance to the fluid motion since not all surfactants exhibit shear elasticity. Under small shear surface viscosity, the interphase will actively impede the development of the divergent flow and can be easily shifted. This can give rise to the vortex motion of the surface layer with practically zero divergence. The type (thermo, concentration-capillary or hydrodynamic) of the flow which produces the fluid motion determines only the intensity of the secondary flow. In our opinion, the presence of random impurities in liquid systems can lead to the formation of similar vortex structures on the surface of emerging gas bubbles and liquid inclusions. For this reason, the efficiency of such flotation, extraction, and other technological processes associated with interfacial mass exchange reduces. Further investigation of this process will improve the effectiveness of these technologies. 00490).

The work was supported by the RFBR-A (project 19-01-

\section{References}

1. G.M. Homsy, E. Meiburg, J. Fluid Mech., 139, 443, (1984)

2. B. Carpenter, G. M. Homsy, J. Fluid Mech. 155. 429 (1986)

3. R. Mei, J.F. Klausner, C.J. Lawrence, Phys. Fluids, 6, 418, (1994)

4. B. Cuenot, J. Magnaudet, B. Spennato, J. Fluid Mech., 339, 25, (1997)

5. R. Palaparthi, D.T. Papageorgiou, C. Maldarelli, J. Fluid Mech., 559, 1, (2006)

6. M. Muradoglu, G. Tryggvason, J. of Computational Phys., 274, 737, (2014)

7. R.L. Merson, J.A. Quinn, A.1.Ch.E. J., 11, 391, (1965)

8. M.J. Vogel, A.H. Hirsa, J. Fluid Mech., 472, 283, (2002)

9. J.M. Lopez, A.H. Hirsa, J. Colloid Interface Sci., 229, $575,(2000)$

10. A.H. Hirsa, J.M. Lopez, R.J. Miraghaie, J. of Fluid Mech., 443, 271, (2001)

11. D.G. Suciu, O. Smigelschi, E. Ruckenstein, AlChE Journal, 13, 1120, (1967)

12. D.G. Suciu, O. Smigelschi, E. Ruckenstein, J. of Colloid and Interface Science, 33, 520, (1970)

13. E. Sada et. al., Chemical Engineering Science, 32, 1171, (1977)

14. T.-C. Wu, Y.-M. Yang, J.-R. Maa, Int. Comm. Heat Mass Transfer, 27, 655, (2000)
15. A. Mizev, Ph. of Fluids, 17, 122107, (2005)

16. M. Roché, Z. Li, I.M. Griffiths, et al., PRL, 112, 208302, (2014)

17. A. Mizev, A. Trofimenko (Shmyrova), D. Schwabe, A. The Euro. Ph. J. Special Topics, 219, 89, (2013)

18. Mizev A.I., Trofimenko (Shmyrova), Fluid Dynamics, 49, 26, (2014)

19. A.I. Mizev, A.V. Shmyrov, 7th Conference of the International Marangoni Association, 96, (2014)

20. D.G. Suciu, O. Smigelschi, E. Ruckenstein, J. Colloid Interface Sci., 88, 520, (1970) 